\title{
Tau Based Therapeutics: Alternative Approaches in the War on Alzheimer's Disease
}

\author{
M. Grundman \\ Correspondance author: Michael Grundman, Global R\&D Partners, LLC and University of California, San Diego, michael@grndpartners.com \\ J Prev Alz Dis 2019;3(6):151-152 \\ Published online April 18, 2019, http:/ / dx.doi.org/10.14283/jpad.2019.13
}

$\mathbf{T}$ This issue of The Journal of Prevention of Alzheimer's Disease highlights EU/US/CTAD TASK FORCE discussions on the topic of taubased therapeutics that were held in association with the 2018 CTAD meeting. The EU/US/CTAD TASK FORCE meeting report (1) is particularly timely given that a number of tau-based therapies are currently being evaluated in clinical trials and will report their results in the not-too-distant future.

The development of tau based therapies for Alzheimer's disease is well justified by scientific research and further supported by practical clinical trial considerations. While abnormalities in abeta metabolism and abeta deposition occur early in $\mathrm{AD}$ there is compelling evidence that cognition in $\mathrm{AD}$ correlates better with tau pathology than with abeta deposition (2). To date, it remains an unresolved question as to whether anti-abeta therapeutics can alter the course of Alzheimer's disease once clinical symptoms appear. While there are hints that this may yet be the case as evidenced by suggestive data on some clinical trial endpoints in the solanezumab phase 3 trial (3) and the aducanumab phase $1 \mathrm{~b}$ study (4) the AD field still awaits definitive proof of efficacy from pivotal trials with antiabeta therapies. Because abeta deposition starts early in the presymptomatic stage of $\mathrm{AD}$, it is possible that antiabeta therapies may be more effective in the preclinical stages of the illness. A number of anti-abeta therapeutic approaches are therefore being studied or have been studied in preclinical AD trials. Pre-clinical AD trials in cognitively normal individuals, however, take a relatively long time to read out (possibly 6 to 8 years) and require more subjects than are required for prodromal and mild AD trials (over 1000 subjects per trial). Recruitment of these trials is also challenging (5). Due to the resources required only a limited number of these important preclinical trials are possible. It is therefore logical and practical to consider tau as an alternative target especially as tau-based approaches may be effective in symptomatic patients. With tau-based approaches it may be possible to demonstrate clinical efficacy in symptomatic patients in whom the rate of disease progression is faster and more predictable. The latter may permit clinical trials to be conducted that are smaller in size, shorter in duration, and less costly. At the same time it should Received February 26, 2019 also be possible to measure the biologic effects of tau interventions directly by measuring changes in tau species in CSF (6), evaluating tau spread with tau PET imaging, and assessing downstream neurodegenerative biomarkers.

Anti-tau approaches also need not be restricted to treatment of patients with Alzheimer's disease. Unlike anti-abeta strategies that may be limited primarily to treatment or prevention of $\mathrm{AD}$ and Down syndrome related dementia (i.e. diseases in which substantial abeta pathology is present), anti-tau approaches may have utility in the treatment of neurodegenerative diseases other than AD in which tau is deposited (e.g. Progressive Supranuclear Palsy (PSP) and Frontotemporal Dementia). Anti-tau antibodies are, in fact, now being evaluated in both AD and PSP clinical trials (1). In addition to anti-tau antibodies and active immunization strategies intended to block tau spreading, tau anti-aggregants, tau kinase inhibitors, and gene therapy approaches that inhibit tau production are also in development.

The EU EU/US/CTAD TASK FORCE meeting report underscores the fact that many questions remain unanswered in the pursuit of tau-based therapeutics. Primary among these questions is which approaches should be prioritized given the abundance and complexity of potential tau targets (7). Which tau epitopes should be targeted? Can targeting only extracellular tau be effective? Would targeting intracellular tau be more useful? Reminiscent of anti-abeta discussions, should the tau endgame be to reduce monomeric tau, tau oligomers, deposited tau fibrils, or some combination of these species? Is it possible that there are consequences to reducing tau too much? These are just some of the concerns that require more data to optimally inform the development of tau-based therapeutics. AD clinical trials will certainly not provide the answers to these questions overnight but they are still likely to be the best source we have for providing the answers to these questions.

\section{References}

1. Cummings J, Blennow K, Johnson K, et al. Anti-Tau Trials for Alzheimer's Disease: A Report from the EU/US/CTAD Task Force. J Prev Alz Dis 2019;3(6):157-163.

2. Ossenkoppele R, Smith R, Ohlsson T, et al. Associations between tau, Abeta and cortical thickness with cognition in Alzheimer disease. Neurology 
2019;92:e601-e12.

3. Honig LS, Vellas B, Woodward M, et al. Trial of Solanezumab for Mild Dementia Due to Alzheimer's Disease. The New England journal of medicine 2018;378:321-30.

4. Sevigny J, Chiao P, Bussiere $\mathrm{T}$, et al. The antibody aducanumab reduces Abeta plaques in Alzheimer's disease. Nature 2016;537:50-6.

5. Rafii MS, Aisen PS. Alzheimer's Disease Clinical Trials: Moving Toward Successful Prevention. CNS drugs 2019;33:99-106.
6. Qureshi IA, Tirucherai G, Ahlijanian MK, Kolaitis G, Bechtold C, Grundman M. A randomized, single ascending dose study of intravenous BIIB092 in healthy participants. Alzheimer's \& dementia 2018;4:746-55.

7. Holtzman DM, Carrillo MC, Hendrix JA, et al. Tau: From research to clinical development. Alzheimer's \& dementia : the journal of the Alzheimer's Association 2016;12:1033-9. 